\title{
Optimal Preview Controller for Linear Discrete-Time Systems: A Virtual System Method
}

\author{
Jiang Wu $(\mathbb{D}$, Huali Zhang $(\mathbb{D}$, and Denggui Fan \\ School of Mathematics and Physics, University of Science and Technology Beijing, Beijing 100083, China \\ Correspondence should be addressed to Jiang Wu; jiangwu@ustb.edu.cn
}

Received 12 April 2021; Revised 13 July 2021; Accepted 21 July 2021; Published 31 July 2021

Academic Editor: Yong-Hong Lan

Copyright (c) 2021 Jiang Wu et al. This is an open access article distributed under the Creative Commons Attribution License, which permits unrestricted use, distribution, and reproduction in any medium, provided the original work is properly cited.

\begin{abstract}
In this paper, a new method for the design of the preview controller for a class of discrete-time systems is proposed based on the virtual system. Firstly, by taking the known future reference signal as the output, the virtual system with similar structures to the controlled system is constructed. Then, the augmented error system is received by translating the controlled system to it and by integrating the error equation. Thus, the tracking problem of the controlled system is transformed into the regulation problem of the augmented error system. Secondly, in view of the minimum principle, the optimal controller of the augmented error system is acquired, and the preview controller of the controlled system is also gained. Further, by discussing the stabilizability and detectability of the augmented error system, the conditions for the existence of the unique positive semidefinite solution to an algebraic Riccati equation are obtained. By using the method in this paper, making difference and dimension expansion for the state equation in designing the augmented error system is avoided and the output can track the reference signals better. Finally, the numerical simulation shows the effectiveness of the proposed controller.
\end{abstract}

\section{Introduction}

When a part of future reference signals or exogenous disturbance signals are known, they can be utilized by preview control and the tracking performance of the closed-loop system can be improved [1]. Because of its good tracking effect, preview control theory has been widely concerned by the academic circle, since it is proposed. After nearly 60 years of development, it has reached many research achievements, such as $H_{\infty}$ preview control [2-4], adaptive preview control [5-7], and information fusion preview control [8-10]. Meanwhile, preview control has also made some progress in the applications of vehicle active suspension system [11-13], robot $[14,15]$ and aircraft [16-18], and other fields.

The augmented error system is a common approach to solve the preview control problem. It is proposed by Tomizuka and Rosenthal [19] in 1979 and has been applied to both discrete-time systems and continuous-time systems. In [19], the augmented error system is constructed by applying difference to both sides of the state equation and to the error equation, which makes the preview tracking problem of the discrete-time system transformed into a standard linear quadratic regulation (LQR) problem. Then, the optimal controller of the augmented error system can be obtained by directly using the existing results. Due to a group of identities about future disturbance signals being added to the augmented error system, the obtained controller for the controlled system contains preview compensation. Based on this technique, Katayama et al. solved the tracking problem of the discrete-time system with preview reference signals in [20], and the conclusion obtained is extended to the condition of continuous-time systems in [21]. In [22], the tracking problem of the continuous-time linear system with both reference signals and disturbance signals previewable is further solved.

In this paper, the method in $[23,24]$ is used to design the optimal preview controller for a class of linear discrete-time systems. In the first place, by using the previewable future reference signal, the virtual system with similar structure to the controlled system is constructed, and the controlled system is translated to the virtual system. Then, the augmented error system is received by combining the after- 
translation system and an equation on the error vector. As a result, the tracking problem of the controlled system is transformed into the regulation problem of the augmented error system. Then, in the process of solving the optimal controller of the augmented error system by the minimum principle, the preview controller of the controlled system is also acquired. Compared with the classical difference method, the method used in this paper does not need to take the difference to the state equation and induce a series of identities on previewable reference signals in the augmented error system, which can make the output chase the reference signals more quickly than that in [20].

The remainder of this paper is organized as follows. In Section 2, the necessary assumptions of the preview control problem are presented. In Section 3, the augmented error system is constructed by introducing the virtual system and an integrator. In Section 4, the preview controller of the controlled system is obtained, and the conditions for the existence of the unique positive semidefinite solution to algebraic Riccati equation are discussed. In Section 5, the effectiveness of the proposed method is verified by a numerical simulation.

\section{Problem Statement}

Consider the following discrete-time linear system:

$$
\left\{\begin{array}{l}
x(k+1)=A x(k)+B u(k) \\
y(k)=C x(k)
\end{array},\right.
$$

where $x(k) \in R^{n}, u(k) \in R^{m}$, and $y(k) \in R^{p}$ are the state vector, the control input, and the output vector, respectively. $A, B$, and $C$ are constant matrices with appropriate dimensions.

Let $r(k) \in R^{p}$ be the reference signal, for which the following assumption is necessary in the preview control problem.

Assumption 1. The preview length of the reference signal is $M_{r}$, which means that at time $k$, the terms $r(k), r(k+1)$, $r(k+2), \ldots, r\left(k+M_{r}\right)$ are available. The future values of the reference signal after time $k+M_{r}$ are constant with $r\left(k+M_{r}\right)$, namely, $r\left(k+M_{r}+j\right)=r\left(k+M_{r}\right), j=1,2,3$, ....

For the coefficient matrices of system (1), the following assumption is needed.

Assumption 2. $(A, B)$ is stabilizable, $(C, A)$ is detectable, and

$$
\operatorname{rank}\left[\begin{array}{cc}
A-I & B \\
C & 0
\end{array}\right]=n+p(\text { full row rank })
$$

Remark 1. If the matrix $\left[\begin{array}{cc}A-I & B \\ C & 0\end{array}\right]$ is of full row rank, the following equation about $\Gamma, \gamma$,

$$
\left\{\begin{array}{l}
(A-I) \Gamma+B \gamma=0 \\
C \Gamma=I
\end{array},\right.
$$

is solvable [23].
The error signal $e(k)$ is defined as the difference between the output and the reference signal, i.e.,

$$
e(k)=y(k)-r(k) .
$$

The purpose of this paper is to design a preview controller so that the output $y(k)$ can track the reference signal $r(k)$ asymptotically.

\section{Derivation of the Augmented Error System}

In order to construct the augmented error system, the virtual system and the integrator need to be introduced.

First of all, by utilizing the known future reference signal, the virtual system is defined. Multiplying $r\left(k+M_{r}\right)$ on both sides of (3), we have

$$
\left\{\begin{array}{l}
(A-I) \Gamma r\left(k+M_{r}\right)+B \gamma r\left(k+M_{r}\right)=0 \\
C \Gamma r\left(k+M_{r}\right)=r\left(k+M_{r}\right)
\end{array}\right.
$$

Let

$$
\begin{aligned}
& x^{*}(k)=\operatorname{\Gamma r}\left(k+M_{r}\right), \\
& u^{*}(k)=\gamma r\left(k+M_{r}\right) .
\end{aligned}
$$

Expressing (5) with $x^{*}(k), u^{*}(k)$ yields

$$
\left\{\begin{array}{l}
x^{*}(k)=A x^{*}(k)+B u^{*}(k) \\
r\left(k+M_{r}\right)=C x^{*}(k)
\end{array}\right.
$$

According to Assumption 1, at time $k$, we have

$$
r\left(k+M_{r}\right)=r\left(k+M_{r}+1\right) \text {. }
$$

So, we can conclude

$$
x^{*}(k)=x^{*}(k+1) .
$$

Thus, (7) can be rewritten as

$$
\left\{\begin{array}{l}
x^{*}(k+1)=A x^{*}(k)+B u^{*}(k) \\
r\left(k+M_{r}\right)=C x^{*}(k)
\end{array}\right.
$$

(10) is the virtual system. Define the following variables:

$$
\left\{\begin{array}{l}
\tilde{x}(k)=x(k)-x^{*}(k) \\
\tilde{u}(k)=u(k)-u^{*}(k) \\
\widetilde{y}(k)=y(k)-C x^{*}(k) \\
\tilde{r}(k)=r(k)-r\left(k+M_{r}\right)
\end{array}\right.
$$

(1) subtracting (10) on both sides, we have

$$
\left\{\begin{array}{l}
\tilde{x}(k+1)=A \tilde{x}(k)+B \tilde{u}(k) \\
\tilde{y}(k)=C \tilde{x}(k)
\end{array}\right.
$$

(12) is the after-translation system.

Secondly, the integrator is introduced. Take $q(k)$ as the sum of error signal $e(k)$ from time $i=0$ to $i=k-1$, namely,

$$
q(k)=\sum_{i=0}^{k-1} e(i) .
$$

So, we have 


$$
\begin{aligned}
q(k+1) & =q(k)+e(k) \\
& =q(k)+C x(k)-r(k) .
\end{aligned}
$$

Because of $r\left(k+M_{r}\right)=C x^{*}(k)$, using the variables in (11) and (14) can be expressed as

$$
q(k+1)=q(k)+C \tilde{x}(k)-\tilde{r}(k) .
$$

Combining (15) and the first equation of (12) yields

$$
X(k+1)=\widetilde{A} X(k)+\widetilde{B} \widetilde{u}(k)+\widetilde{D} \widetilde{r}(k),
$$

where

$$
\begin{aligned}
X(k) & =\left[\begin{array}{c}
q(k) \\
\tilde{x}(k)
\end{array}\right], \\
\widetilde{A} & =\left[\begin{array}{cc}
I & C \\
0 & A
\end{array}\right], \\
\widetilde{B} & =\left[\begin{array}{c}
0 \\
B
\end{array}\right], \\
\widetilde{D} & =\left[\begin{array}{c}
-I \\
0
\end{array}\right] .
\end{aligned}
$$

Choose the output equation of system (16) as

$$
q(k)=\widetilde{C} X(k),
$$

where $\widetilde{C}=\left[\begin{array}{ll}I & 0\end{array}\right]$. Then, we have

$$
\left\{\begin{array}{l}
X(k+1)=\widetilde{A} X(k)+\widetilde{B} \widetilde{u}(k)+\widetilde{D} \widetilde{r}(k) \\
q(k)=\widetilde{C} X(k)
\end{array}\right.
$$

(19) is the augmented error system.

In order to achieve the control purpose, the performance index can be defined as follows:

$$
J=\frac{1}{2} \sum_{k=0}^{\infty}\left[q^{T}(k) Q_{q} q(k)+\tilde{u}^{T}(k) R \tilde{u}(k)\right],
$$

where the weight matrices satisfy $Q_{q}>0, R>0$.

Remark 2. It is reasonable to take (20) as the performance index function because when $\lim _{k \longrightarrow \infty} q(k)=0$, we have $\lim _{k \rightarrow \infty} e(k)=0$.

Further, expressing the performance index (20) with the related variables in system (19), we can get

$$
J=\frac{1}{2} \sum_{k=0}^{\infty}\left[X^{T}(k) Q X(k)+\tilde{u}^{T}(k) R \widetilde{u}(k)\right],
$$

where $Q=\widetilde{C}^{T} Q_{q} \widetilde{C}$. In this way, the tracking problem of the controlled system is transformed into the optimal regulation problem of system (19) under the performance index function (21).

\section{Design of the Preview Controller}

First, the optimal controller of the augmented error system (19) is solved.

Due to the term $\widetilde{D} \widetilde{r}(k)$ contained in (19), the conclusion of the optimal regulation problem in infinite time cannot be applied to the augmented error system to derivate the optimal controller directly. Therefore, the finite-time horizon condition is considered at first. It is natural that the corresponding performance index should be taken as

$$
\widetilde{J}=\frac{1}{2} \sum_{k=0}^{N}\left[X^{T}(k) Q X(k)+\widetilde{u}^{T}(k) R \widetilde{u}(k)\right],
$$

where $N$ is the terminal time. Let Hamilton function be

$$
H=\frac{1}{2}\left[X^{T}(k) Q X(k)+\widetilde{u}^{T}(k) R \widetilde{u}(k)\right]+\lambda^{T}(k+1)[\widetilde{A} X(k)+\widetilde{B} \widetilde{u}(k)+\widetilde{D} \widetilde{r}(k)]
$$

where $\lambda(k+1)$ is the costate vector with the same dimensions of $X(k)$.

The optimal control input can be solved by the control equation $(\partial H / \partial \widetilde{u}(k))=0$, and the corresponding optimal state vector and costate vector satisfy the regular equation:

$$
\begin{aligned}
X(k+1) & =\frac{\partial H}{\partial \lambda(k+1)}, \\
\lambda(k) & =\frac{\partial H}{\partial X(k)},
\end{aligned}
$$

with the boundary conditions

$$
\begin{aligned}
X(0) & =\left[\begin{array}{l}
q(0) \\
\tilde{x}(0)
\end{array}\right], \\
\lambda(N+1) & =0 .
\end{aligned}
$$

So, we have

$$
\left\{\begin{array}{l}
\widetilde{u}(k)=-R^{-1} \widetilde{B}^{T} \lambda(k+1) \\
X(k+1)=\widetilde{A} X(k)+\widetilde{B} \tilde{u}(k)+\widetilde{D} \tilde{r}(k) \\
\lambda(k)=Q X(k)+\widetilde{A}^{T} \lambda(k+1) \\
X(0)=\left[\begin{array}{l}
q(0) \\
\tilde{x}(0)
\end{array}\right] \\
\lambda(N+1)=0
\end{array}\right.
$$

Then, $\tilde{u}(t)$ is solved. Similar to the continuous-time system condition in [23], suppose $\lambda(k)$ has the form of

$$
\lambda(k)=P(k) X(k)+g(k) .
$$

Substituting (27) and the first equation of (26) into the second and the third equations of (26) yields 


$$
\left\{\begin{array}{l}
X(k+1)=\widetilde{A} X(k)-\widetilde{B} R^{-1} \widetilde{B}^{T} P(k+1) X(k+1)-\widetilde{B} R^{-1} \widetilde{B}^{T} g(k+1)+\widetilde{D} \widetilde{r}(k) \\
\lambda(k)=Q X(k)+\widetilde{A}^{T} P(k+1) X(k+1)+\widetilde{A}^{T} g(k+1)
\end{array}\right.
$$

According to the first equation of (28), we can get

$$
\left[I+\widetilde{B} R^{-1} \widetilde{B}^{T} P(k+1)\right] X(k+1)=\widetilde{A} X(k)-\widetilde{B} R^{-1} \widetilde{B}^{T} g(k+1)+\widetilde{D} \widetilde{r}(k)
$$

From [25], we know that if $P(k+1)$ is a positive semidefinite matrix, then the matrix $I+\widetilde{B} R^{-1} \widetilde{B}^{T} P(k+1)$ is invertible and

$$
\left[I+\widetilde{B} R^{-1} \widetilde{B}^{T} P(k+1)\right]^{-1}=I-\widetilde{B}\left[R+\widetilde{B}^{T} P(k+1) \widetilde{B}\right]^{-1} \widetilde{B}^{T} P(k+1) .
$$

Thus, (29) can be rewritten as

$$
\begin{aligned}
X(k+1)= & {\left[I+\widetilde{B} R^{-1} \widetilde{B}^{T} P(k+1)\right]^{-1} \widetilde{A} X(k) } \\
& -\left[I+\widetilde{B} R^{-1} \widetilde{B}^{T} P(k+1)\right]^{-1} \widetilde{B} R^{-1} \widetilde{B}^{T} g(k+1) \\
& +\left[I+\widetilde{B} R^{-1} \widetilde{B}^{T} P(k+1)\right]^{-1} \widetilde{D} \widetilde{r}(k) .
\end{aligned}
$$

Substituting (31) into the second equation of (28), we can obtain

$$
\begin{aligned}
\lambda(k)= & {\left[Q+\widetilde{A}^{T} P(k+1)\left[I+\widetilde{B} R^{-1} \widetilde{B}^{T} P(k+1)\right]^{-1} \widetilde{A}\right] X(k) } \\
& +\left[\widetilde{A}^{T}-\widetilde{A}^{T} P(k+1)\left[I+\widetilde{B} R^{-1} \widetilde{B}^{T} P(k+1)\right]^{-1} \widetilde{B} R^{-1} \widetilde{B}^{T}\right] g(k+1) \\
& +\widetilde{A}^{T} P(k+1)\left[I+\widetilde{B} R^{-1} \widetilde{B}^{T} P(k+1)\right]^{-1} \widetilde{D} \widetilde{r}(k)
\end{aligned}
$$

Comparing the coefficient matrices of (27) and (32), we can conclude

$$
\begin{aligned}
P(k)= & Q+\widetilde{A}^{T} P(k+1)\left[I+\widetilde{B} R^{-1} \widetilde{B}^{T} P(k+1)\right]^{-1} \widetilde{A}, \\
g(k)= & {\left[\widetilde{A}^{T}-\widetilde{A}^{T} P(k+1)\left[I+\widetilde{B} R^{-1} \widetilde{B}^{T} P(k+1)\right]^{-1} \widetilde{B} R^{-1} \widetilde{B}^{T}\right] g(k+1) } \\
& +\widetilde{A}^{T} P(k+1)\left[I+\widetilde{B} R^{-1} \widetilde{B}^{T} P(k+1)\right]^{-1} \widetilde{D} \widetilde{r}(k) .
\end{aligned}
$$

Substituting (30) into (33), we can get 


$$
P(k)=Q+\widetilde{A}^{T} P(k+1) \widetilde{A}-\widetilde{A}^{T} P(k+1) \widetilde{B}\left[R+\widetilde{B}^{T} P(k+1) \widetilde{B}\right]^{-1} \widetilde{B}^{T} P(k+1) \widetilde{A} .
$$

(35) is a Riccati difference equation. According to (27) and the boundary condition $\lambda(N+1)=0$, the termination conditions of (34) and (35) are

$$
\begin{aligned}
& g(N+1)=0, \\
& P(N+1)=0 .
\end{aligned}
$$

Let $N \longrightarrow \infty$. According to [26], if $(\widetilde{A}, \widetilde{B})$ is stabilizable and $\left(Q^{1 / 2}, \widetilde{A}\right)$ is detectable, the solution $P(k)$ of $(35)$ converges to a constant matrix $P$, where $P$ is the unique positive semidefinite solution of the algebraic Riccati equation:

$$
P=Q+\widetilde{A}^{T} P \widetilde{A}-\widetilde{A}^{T} P \widetilde{B}\left[R+\widetilde{B}^{T} P \widetilde{B}\right]^{-1} \widetilde{B}^{T} P \widetilde{A} .
$$

As a result, for (34), $g(k)$ can be denoted as

$$
g(k)=S_{1} g(k+1)+S_{2} \widetilde{r}(k),
$$

where

$$
\begin{gathered}
S_{1}=\widetilde{A}^{T}-\widetilde{A}^{T} P\left[I+\widetilde{B} R^{-1} \widetilde{B}^{T} P\right]^{-1} \widetilde{B} R^{-1} \widetilde{B}^{T}, \\
S_{2}=\widetilde{A}^{T} P\left[I+\widetilde{B} R^{-1} \widetilde{B}^{T} P\right]^{-1} \widetilde{D} .
\end{gathered}
$$

According to Assumption 1, when $i \geq M_{r}, r(k+i)=$ $r\left(k+M_{r}\right)$. Consequently, we have $\widetilde{r}(k+i)=0\left(i \geq M_{r}\right)$. If $g(k)$ has the form of

$$
g(k)=\sum_{i=0}^{M_{r}-1} F_{r}(i) \widetilde{r}(k+i)
$$

where $F_{r}(i),\left(i=0,1,2, \ldots, M_{r}-1\right)$ are weight matrices, then (38) can be written as

$$
\sum_{i=0}^{M_{r}-1} F_{r}(i) \widetilde{r}(k+i)=S_{1} \sum_{i=0}^{M_{r}-1} F_{r}(i) \widetilde{r}(k+1+i)+S_{2} \widetilde{r}(k),
$$

and $F_{r}(i)\left(i=0,1,2, \ldots, M_{r}-1\right)$ can be solved by the following equations:

$$
\left\{\begin{array}{l}
F_{r}(0)=S_{2} \\
F_{r}(1)=S_{1} F_{r}(0)=S_{1} S_{2} \\
F_{r}(2)=S_{1} F_{r}(1)=S_{1}^{2} S_{2} \\
\vdots \\
F_{r}\left(M_{r}-2\right)=S_{1} F_{r}\left(M_{r}-1\right)=S_{1}^{M_{r}-2} S_{2} \\
F_{r}\left(M_{r}-1\right)=S_{1} F_{r}\left(M_{r}-2\right)=S_{1}^{M_{r}-1} S_{2}
\end{array}\right.
$$

Denote (43) as

$$
F_{r}(i)=S_{1}^{i} S_{2} \quad\left(i=0,1,2, \ldots, M_{r}-1\right) .
$$

Substituting (44) into (41) yields

$$
g(k)=\sum_{i=0}^{M_{r}-1} S_{1}^{i} S_{2} \widetilde{r}(k+i)
$$

Substituting (27), (31), and (45) into the first equation of (26), we can get

$$
\widetilde{u}(k)=K X(k)+\sum_{i=0}^{M_{r}} K_{r}(i) \widetilde{r}(k+i)
$$

where

$$
\begin{aligned}
K & =-\left[R+\widetilde{B}^{T} P \widetilde{B}\right]^{-1} \widetilde{B}^{T} P \widetilde{A} \\
K_{r}(0) & =-\left[R+\widetilde{B}^{T} P \widetilde{B}\right]^{-1} \widetilde{B}^{T} P \widetilde{D} \\
K_{r}(i) & =-\left[R+\widetilde{B}^{T} P \widetilde{B}\right]^{-1} \widetilde{B}^{T} S_{1}^{i-1} S_{2} \quad\left(i=1,2, \ldots, M_{r}\right) .
\end{aligned}
$$

Then, we have the following theorem.

Theorem 1. If Assumption 1 holds, $(\widetilde{A}, \widetilde{B})$ is stabilizable and $\left(Q^{1 / 2}, \widetilde{A}\right)$ is detectable, the optimal controller of system (19) under the performance index (21) is

$$
\widetilde{u}(k)=K X(k)+\sum_{i=0}^{M_{r}} K_{r}(i) \widetilde{r}(k+i),
$$

where $P$ satisfies the algebraic Riccati equation (37), $K, K_{r}(i)\left(i=0,1,2, \ldots, M_{r}\right)$ meet (47)-(49), and $S_{1}, S_{2}$ meet (39) and (40).

In order to get the preview controller for system (1), the following lemma is needed.

Lemma 1 (see [26]). ( $\widetilde{A}, \widetilde{B})$ is stabilizable if and only if $(A, B)$ is stabilizable and $\left[\begin{array}{cc}A-I & B \\ C & 0\end{array}\right]$ is of full row rank; $\left(Q^{1 / 2}, \widetilde{A}\right)$ is detectable if and only if $(C, A)$ is detectable and $Q_{q}$ is a positive definite matrix.

Let $K$ be blocked into

$$
K=\left[K_{q}, K_{x}\right],
$$

where $K_{q}$ and $K_{x}$ are matrices, corresponding to $X(k)=\left[\begin{array}{c}q(k) \\ \tilde{x}(k)\end{array}\right]$. Thus, (50) can be rewritten as

$$
\widetilde{u}(k)=K_{q} q(k)+K_{x} \tilde{x}(k)+\sum_{i=0}^{M_{r}} K_{r}(i) \widetilde{r}(k+i) .
$$

On the basis of (6), (11), and (13), the following theorem about the preview controller of system (1) can be obtained.

Theorem 2. If Assumptions 1 and 2 hold, the preview controller of system (1) with the performance index (20) is 


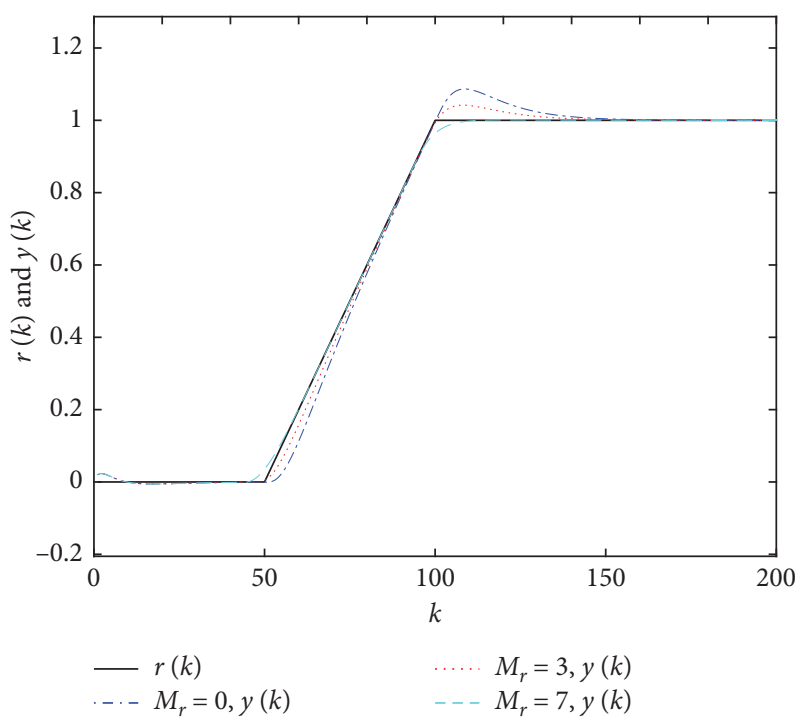

FIGURE 1: The output responses of system (1) with preview lengths $M_{r}=0, M_{r}=3$, and $M_{r}=7$, respectively.

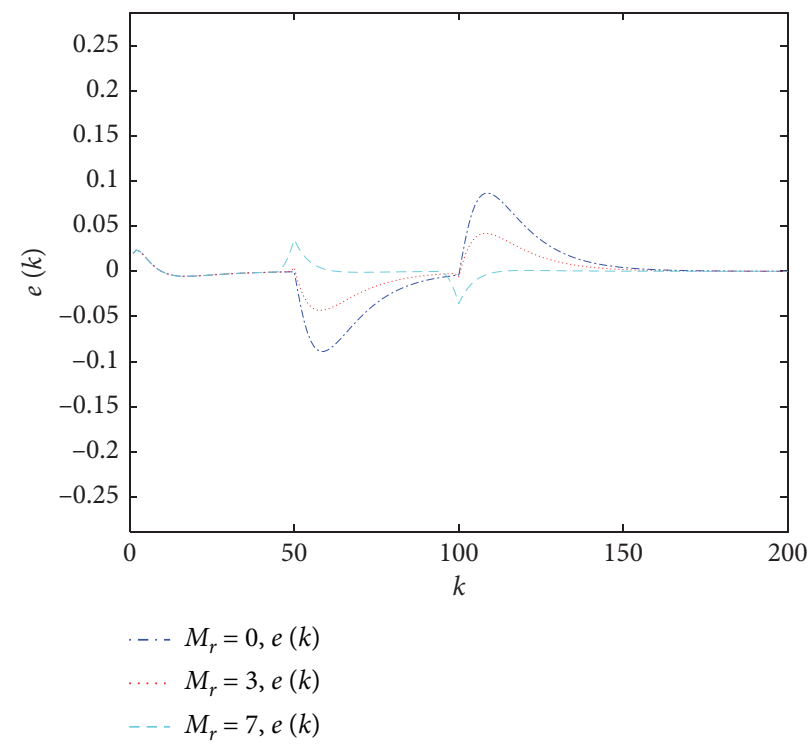

FiguRe 2: The tracking errors of system (1) with preview lengths $M_{r}=0, M_{r}=3$, and $M_{r}=7$, respectively.

$$
\begin{aligned}
u(k)= & K_{q} \sum_{i=0}^{k-1} e(i)+K_{x} x(k)+\sum_{i=0}^{M_{r}} K_{r}(i)\left[r(k+i)-r\left(k+M_{r}+i\right)\right] \\
& -K_{x} \operatorname{\Gamma r}\left(k+M_{r}\right)+\gamma r\left(k+M_{r}\right),
\end{aligned}
$$

where $P$ is the unique positive semidefinite solution, satisfying the algebraic Riccati equation (37), $K, K_{r}(i)(i=$ $0,1,2, \ldots, M_{r}$ ) meet (47)-(49), and $S_{1}, S_{2}$ meet (39) and (40).

It can be seen from (52) that the optimal preview controller of system (1) consists of four parts. The first part is the sum of tracking errors term $K_{q} \sum_{i=0}^{k-1} e(i)$, which is originated from the employment of the integrator. The second part is the state feedback $K_{x} x(k)$. The third part is the preview compensation of reference signals $\sum_{i=0}^{M_{r}} K_{r}(i)\left[r(k+i)-r\left(k+M_{r}+i\right)\right]$. The last one $-K_{x} \Gamma r(k+$ $\left.M_{r}\right)+\gamma r\left(k+M_{r}\right)$ is the preview complement from the virtual system. 


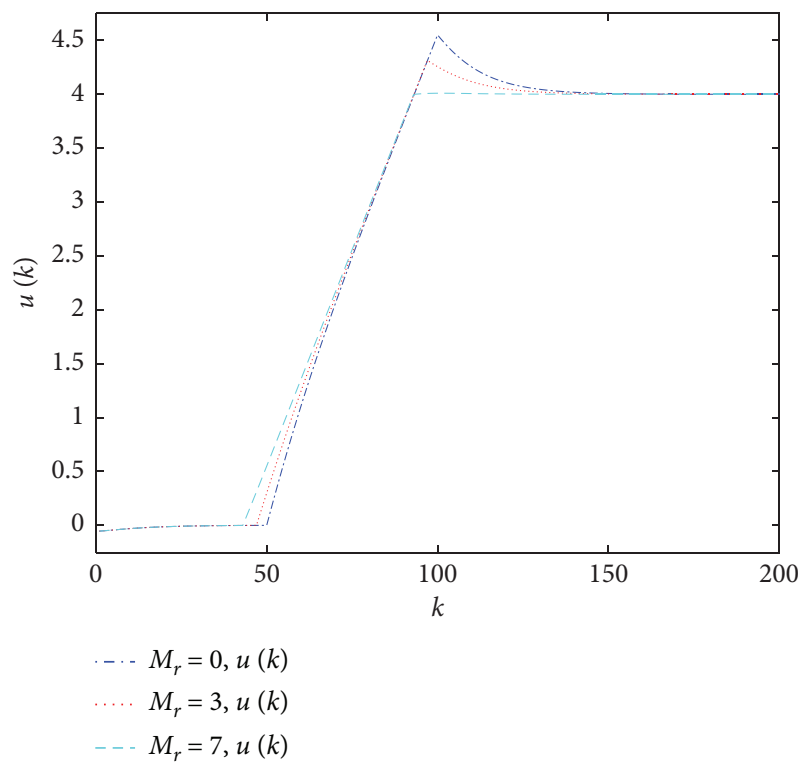

Figure 3: The control inputs of system (1) with preview lengths $M_{r}=0, M_{r}=3$, and $M_{r}=7$, respectively.

\section{Numerical Example}

Consider system (1) with coefficient matrices

$$
\begin{aligned}
& A=\left[\begin{array}{ll}
0.8 & 1 \\
0 & 0.6
\end{array}\right], \\
& B=\left[\begin{array}{l}
-0.1 \\
0.2
\end{array}\right], \\
& C=\left[\begin{array}{ll}
0.1 & 0.1
\end{array}\right] .
\end{aligned}
$$

Let the initial state be $x(0)=\left[\begin{array}{ll}0.1 & 0.1\end{array}\right]^{T}$ and the reference signal be

$$
r(k)= \begin{cases}0 & 0 \leq k<50 \\ 0.02(k-50) & 50 \leq k<100 \\ 1 & k \geq 100\end{cases}
$$

Choose the weight matrices in the performance index function (20),

$$
Q_{q}=1, R=10 .
$$
follows:

The tracking performance of the closed-loop system is as

Figure 1 shows the output responses of the system when the preview lengths of the reference signal are $M_{r}=0, M_{r}=3$, and $M_{r}=7$, respectively. Figures 2 and 3 show the tracking errors and the control inputs with different preview lengths, respectively. It can be seen from Figures 1 and 2 that along with the increase of the preview length, the system output can track the reference signal in advance and the overshoot and the average error can gradually decrease. Figure 3 shows that the requirements of the limit performance to the controller can be effectively reduced by employing the preview compensation.
Unlike the condition in [20], during the ramp section, the output in this paper chases the reference signal more quickly, which means that when the preview length is insufficient, the method in this paper is more effective.

\section{Conclusion}

In this paper, the preview controller is designed for a class of linear discrete-time systems by introducing the virtual system and the integrator. When constructing the augmented error system, there is no need to take the difference to the state equation and to add a series of identities on preview reference signals to the augmented error system. The numerical simulation example shows the proposed preview controller can track the reference signals more effectively than the existing results in some conditions.

\section{Data Availability}

The data used to support the findings of this study are included within the article.

\section{Conflicts of Interest}

The authors declare that they have no conflicts of interest.

\section{Acknowledgments}

This research was supported by the National Science Foundation of China (Grant no. 12072021) and the Fundamental Research Funds for the Central Universities (FRFTP-20-013A3).

\section{References}

[1] T. Tsuchiya and T. Egami, Digital Preview and Predictive Control, Enterprise Press, Tokyo, Japan, 1992.

[2] A. Hazell and D. J. N. Limebeer, "An efficient algorithm for discrete-time Ho preview control," Automatica, vol. 44, no. 9, pp. 2441-2448, 2008.

[3] A. Kojima, " $H_{\infty}$ controller design for preview and delayed systems," IEEE Transactions on Automatic Control, vol. 60, no. 2, pp. 404-419, 2015.

[4] H. Wang, M. Fu, and H. Zhang, " $H_{\infty}$ control for stochastic systems with disturbance preview," SIAM Journal on Control and Optimization, vol. 58, no. 1, pp. 215-242, 2020.

[5] N. Birla and A. Swarup, "Adaptive preview control: a novel control structure," IFAC Proceedings Volumes, vol. 45, no. 1, pp. 13-16, 2012.

[6] D. Wang, F. Liao, and M. Tomizuka, "Adaptive preview control for piecewise discrete-time systems using multiple models," Applied Mathematical Modelling, vol. 40, no. 23-24, pp. 9932-9946, 2016.

[7] C.-E. Ren and C. P. Chen, "Adaptive preview consensus control for discrete-time nonlinear multi-agent systems with unknown control directions," Transactions of the Institute of Measurement and Control, vol. 42, no. 15, pp. 2941-2950, 2020.

[8] Z. Zhen, Z. Wang, and D. Wang, "Optimal preview tracking control based on information fusion in error system," Control Theory and Applications, vol. 26, no. 4, pp. 425-428, 2009. 
[9] Z.-Y. Zhen, Z.-S. Wang, and D.-B. Wang, "Information fusion estimation based preview control for discrete linear system," Acta Automatica Sinica, vol. 36, no. 2, pp. 347-352, 2010.

[10] C. Gao, B. Zhao, and Z. Hu, "Linear discrete information fusion preview control algorithm based on the observer," Aerospace Control, vol. 36, no. 5, pp. 9-24, 2018.

[11] J. Zhao, X. Hua, Y. Cao, L. Fan, X. Mei, and Z. Xie, "Design of an integrated controller for active suspension systems based on wheelbase preview and wavelet noise filter," Journal of Intelligent \& Fuzzy Systems, vol. 36, no. 4, pp. 3911-3921, 2019.

[12] J. N. Strohm and F. Christ, "Preview $H_{\infty}$ control of a hybrid suspension system," IFAC-Papers OnLine, vol. 52, no. 5, pp. 237-242, 2019.

[13] B.-s. Kwon, D. Kang, and K. Yi, "Wheelbase preview control of an active suspension with a disturbance-decoupled observer to improve vehicle ride comfort," Proceedings of the Institution of Mechanical Engineers, Part D: Journal of Automobile Engineering, vol. 234, no. 6, pp. 1725-1745, 2020.

[14] K. Al Khudir, G. Halvorsen, L. Lanari, and A. De Luca, "Stable torque optimization for redundant robots using a short preview," IEEE Robotics and Automation Letters, vol. 4, no. 2, pp. 2046-2053, 2019.

[15] K. Ryu, J. Yoo, J. Back, and I.-W. Park, "Preview control-based online walking pattern generation for biped robots with vertical center-of-mass motion," International Journal of Precision Engineering and Manufacturing, vol. 21, no. 9, pp. 1653-1661, 2020.

[16] H. Tokutake, S. Okada, and S. Sunada, "Disturbance preview controller and its application to a small UAV," Transactions of the Japan Society for Aeronautical and Space Sciences, vol. 55, no. 1, pp. 76-78, 2012.

[17] D. Uchida, T. Shimomura, and Y. Hamada, "Aircraft gust alleviation preview control with a discrete-time LPV model in consideration of the elastic mode," IFAC-Papers OnLine, vol. 52, no. 28, pp. 184-189, 2019.

[18] Y. Liu, H. Wang, and J. Fan, "Novel docking controller for autonomous aerial refueling with probe direct control and learning-based preview method," Aerospace Science and Technology, vol. 94, p. 14, Article ID 105403, 2019.

[19] M. Tomizuka and D. E. Rosenthal, "On the optimal digital state vector feedback controller with integral and preview actions," Journal of Dynamic Systems, Measurement, and Control, vol. 101, no. 2, pp. 172-178, 1979.

[20] T. Katayama, T. Ohki, T. Inoue, and T. Kato, "Design of an optimal controller for a discrete-time system subject to previewable demand," International Journal of Control, vol. 41, no. 3, pp. 677-699, 1985.

[21] T. Katayama and T. Hirono, "Design of an optimal servomechanism with preview action and its dual problem," International Journal of Control, vol. 45, no. 2, pp. 407-420, 1987.

[22] F. Liao, Y. Tang, H. Liu, and Y. Wang, "Design of an optimal preview controller for continuous-time systems," International Journal of Wavelets, Multiresolution and Information Processing, vol. 9, no. 4, pp. 655-673, 2011.

[23] J. Wu, F. Liao, and M. Tomizuka, "Optimal preview control for a linear continuous-time stochastic control system in finite-time horizon," International Journal of Systems Science, vol. 48, no. 1, pp. 129-137, 2017.

[24] J. Wu, F. Liao, and Z. Xu, "Preview control for a class of linear stochastic systems with multiplicative noise," International Journal of Systems Science, vol. 50, no. 14, pp. 2592-2603, 2019.
[25] K. Ogata, Discrete-time Control Systems, Prentice Hall, Upper Saddle River, NJ, USA, Second edition, 1995.

[26] F. Liao and H. Liu, "Design of an optimal preview controller for a kind of discrete-time systems," Journal of University of Science and Technology Beijing, vol. 29, no. 5, pp. 542-547, 2007. 